Bangladesh J. Plant Taxon. 20(2): 145-162, 2013 (December)

(C) 2013 Bangladesh Association of Plant Taxonomists

\title{
DIVERSITY IN ANGIOSPERM FLORA OF TEKNAF WILDLIFE SANCTUARY, BANGLADESH
}

\author{
Mohammad Zashim Uddin ${ }^{1}$, Md. FaKhruL Alam, Md. Abdur Rhaman ${ }^{2}$ AND \\ MD. ABUL Hassan \\ Department of Botany, University of Dhaka, Dhaka-1000, Bangladesh
}

Keywords: Angiosperm diversity; Teknaf wildlife sanctuary; Bangladesh.

\begin{abstract}
Teknaf Wildlife Sanctuary has been explored to assess angioperm diversity using traditional taxonomic techniques during 2010 to 2011. The assessment has resulted in recording of total 535 angioperm species under 103 familiies and 370 genera. For each species scientific name, Bangla name (whenever available), family and habit are provided. Of 535 species, 178 represented by herbs, 110 by shrubs, 150 by trees, 87 by climbers and 10 by epiphytes. In Magnoliopsida (dicots), Fabaceae is the largest family represented by 38 species, while in Liliopsida (monocots), Poaceae is the largest family represented by 29 species.
\end{abstract}

\section{Introduction}

Teknaf Wildlife Sanctuary, previously declared as game reserve in 1983 under the Bangladesh Wildlife (Preservation) (Amendment Act, 1974), is located in the Teknaf and Ukhia Upazilas of Cox's Bazar district near Myanmar border. Geographical position of the reserve is in between $20^{\circ} 52^{\prime \prime}-21^{0} 09^{\prime \prime} \mathrm{N}$ and $92^{0} 08^{\prime \prime}-92^{0} 18^{\prime \prime} \mathrm{E}$ (Rosario, 1997). The Reserve is bordered by the Bay of Bengal to the south and west, the Naf River to the east and Monkhali and Thainkhali to the north. The reserve is locally managed by three range offices (Teknaf, Whykhong and Shilkhali ranges) and ten forest bits. The total area of the reserve is about 11651 ha (Green, 1987).

Teknaf wildlife sanctuary in past supported mixed evergreen and semi-evergreen forests which over the period have been substantially altered due to heavy biotic pressure. The topography of sanctuary is very undulating and covered with a linear hill range (elongation north to south reaching an altitude up to $700 \mathrm{~m}$ ), gently sloping to rugged hills and cliffs running down the central part of the peninsula, with a north-south length of nearly $28 \mathrm{~km}$ and an east-west width of 3-5 km. The range has several projections running towards east and west and interspersed by valleys, gullies and streams. These are crossed by numerous streams flowing down to the Naf river in the east and the Bay of Bengal in the west. Most of the streams are seasonal and dry up during off-monsoon season. The hills of the Sanctuary are composed of upper tertiary rocks (Pliocene and Miocene epoch) with 3 representative geological series: Surma, Tipam and Dhupitila (Choudhury, 1969). The soils vary from clay to clayey loam on level ground, and from sandy loam to coarse sand on hilly land (Choudhury, 1969). The sanctuary area enjoys a moist tropical maritime climate and rainfall is frequent and heavy during the monsoon season (May to October) ranging between $130 \mathrm{~mm}$ to $940 \mathrm{~mm}$. Temperature ranges from $15^{\circ} \mathrm{C}$ to $32^{\circ} \mathrm{C}$, whereas humidity ranges from $27 \%$ to $99 \%$ (BBS, 2011).

As the Government commitment to the Convention on Biological Diversity (CBD), Taxonomists have already started to assess and document the floral diversity of different protected areas of Bangladesh. There are a few reports available on the Teknaf Wildlife Sanctuary. A list of

\footnotetext{
${ }^{1}$ Corresponding author, Email: zashim07@yahoo.com

${ }^{2}$ ACF, Teknaf Wildlife Sanctuary
} 
important timber yielding plants of the Teknaf game reserve has been mentioned in the forestry master plan of Cox's Bazar south division (Cowan, 1923). Many years later, Khan et al. (1994) has made an assessment work on Teknaf game reserve to focus on ecologically and economically important plant species. Although co-management issues of Teknaf game reserve have been studied by several authors (Bari and Dutta, 2004; Molla et al., 2004), little is known about the floral diversity of the sanctuary. The sanctuary is very rich in flora and represents different ecosystems including hill forest, mangrove formation and sandune. Conservation significance of the sanctuary is also high because of the presence of Asian threatened elephant population. Currently floral diversity of the sanctuary is under threat due to various pressure including anthropogenic activities and the presence of Rohynga refugee along the eastern border. For making proper conservation and management plans of the sanctuary before its complete degradation, data on the floral diversity is essential. In order to provide such information, in the present study an attempt has been made to document diversity of angiosperm flora of Teknaf Wildlife Sanctuary.

\section{Materials and Methods}

Specimen collections have been made in the Teknaf wildlife sanctuary at 2-months intervals between November 2010 and December 2011. The collections covered all habitats of the study area including hilltops, slopes, foothills, valleys, sand dune and wet areas including mangrove stretch along the Naf river. Special attention has been given to locate the species already listed as threatened categories in the country. Fertile plant specimens (flowering or fruiting specimens) were collected and processed using standard herbarium techniques (Hyland, 1972; Alexiades, 1996). The plant specimens were identified by consulting different Floras and literature, viz., Hooker (1872-1897), Prain (1903), Uddin and Hassan (2004), Siddiqui et al. (2007) and Ahmed et al. (2008a, 2009a), and by comparing with the herbarium specimens available at Dhaka University Salar Khan Herbarium (DUSH) and Bangladesh National Herbarium (DACB). For updated nomenclature of the species, Siddiqui et al. (2007) and Ahmed et al. (2008a,b, 2009a,b,c) were followed. Threatened categories of plants were confirmed with the help of Khan et al. (2001). Some noxious exotic plant species were also identified comparing with the reports of Islam et al. (2003), Hossain and Pasha (2004) and Akter and Zuberi (2009). Cronquist (1981) system of classification has been followed to determine the families. Voucher specimens are deposited at DUSH.

\section{Results and Discussion}

A total of 535 angiosperm species (wild and cultivated) have been identified and presented based on the present study (collection and observation) in the Teknaf Wildlife Sanctuary. These species have been assigned to 103 families and 370 genera. For each species scientific name, Bangla name (when available) and family name are provided (Table 1). $46 \%$ of the total species are represented by 15 families, whereas the rest $54 \%$ by 87 families. In Magnoliopsida (dicots) Fabaceae is the largest family represented by 38 species, while in Liliopsida (monocots) Poaceae is the largest family represented by 29 species. Each of 31 families is represented by only one species. Of 535 species recorded here, herbs are represented by 178 species, 110 by shrubs, 150 by trees, 87 by climbers and 10 by epiphytes including parasites. Nineteen species including one gymnosperm (Gnetum oblongum) listed as threatened in the Red Data Book of the country have also been detected in this sanctuary (Khan et al., 2001).

Once, the Teknaf area has been classified as hill forest dominated by evergreen and deciduous trees. Currently maximum area of the forest has been degraded and denuded and exposed to sun. 
In such a situation some natural and planted forest covers have been observed in Shilkhali, Shaplapur, Kudumgoha and Muchani along the road to Teknaf range office. The most common first canopy tree species observed in this area are Dipterocarpus turbinatus (Teliagarjan), $D$. alatus (Duliagarjan,) Hopea odorata (Telsur), Artocarpus chaplasha (Chapalish), Tetrameles nudiflora (Chundul), Anisoptera scaphula (Bailum), Syzygium firmum (Dhakijam), Mangifera sylvatica (Uriam) and Swintonia floribunda (Civit). The second storey is dominated by Bursera serrata, Artocarpus lakucha, Brownlowia alata, Anogeissus acuminata, Alstonia scholaris, Albizia procera, Neolamarckia cadamba, Ficus altissima, Syzygium cumini, Terminalia bellirica, Garuga pinnata, Elaeocarpus floribunda, Lagerstroemia speciosa, Toona ciliata, Dillenia pentagyna, Aphanamixis polystachya, Gmelina arborea, Zanthoxylum rhetsa, Quercus velutina, Stereospermum personatum, Vitex peduncularis, Sterculia villosa, Bombax insigne, Anacardium occidentale and Ficus infectoria. The third storey of the forest is dominated by Syzygium fruticosum, Suregada multiflora, Streblus asper, Grewia microcos, Mallotus philippensis, Litsea glutinosa, Pterospermum semisagittatum, Tabernaemontana recurvata, Erioglossum edulis, Glochidion multiloculare, Saraca indica, Callicarpa arborea, Ficus hispida, F. semicordata, Maesa indica, Flacourtia indica, Micromelum minutum, Phyllanthus reticulatus, Pterospermum semisagittatum, Premna esculenta, Dalbergia volubilis, Randia dumetorum, Woodfordia fruticosa, Litsea monopetala and Antidesma ghaesembilla.

The most common species found as the forest undergrowth are mostly the members of Acanthaceae, Araceae, Asteraceae, Cyperaceae, Euphobiaceae, Fabaceae, Poaceae, Rubiaceae and Zingiberaceae. In the degraded area of the hills where tree cover is almost absent, the most common species are Clerodendrum viscosum, Chromolaena odorata, Mimosa pudica, Urena lobata, Melastoma malabathricum, Borreria hispida, Triumfettra rhomboidea, Ixora javanica, Ageratum conyzoides, Mikania cordata, Osbeckia asperculis, Globba multiflora, Desmodium triquetrum, Crotolaria juncea, Leea crispa, Sida acuta, Ocimum americanum, Hyptis sauveolens and also some members of common climber families including Dioscoriaceae, Combretaceae, Vitaceae, Convolvulaceae, Menispermaceae, Apocynaceae, Asclepiadaceae and Cucurbitaceae. Relatively wet areas are dominated by Hydrolea zeylanica, Ludwigia adscendens, Ipomoea aquatica, Alternanthera sessilis, Tilanthera philoxeroides, Monochoria vaginalis, Colocasia esculenta, Hedyotis scandens, Eichhornia crassipes, and also the member's sedge, grass, aroids.

In the edge of forest and on the bank of streams the most common climbers are Mikania cordata, Ichnocarpus frutescens, Merremia umbellata, Calycopteris floribunda, Derris trifoliata, Thunbergia grandiflora, Cyclea barbata, Cissus adnata, Cayratia japonica, Stephania harnadifolia, Combretum apetalum, Acacia concinna, Vitis repens, Bridelia secandens, Smilax zeylanica, Dioscorea pentaphylla, Dioscorea bulbifera, Hoya parasitica and Argyreia capitiformis. Large canopy trees are special habitats for epiphyte and parasites. The most common epiphytes are Aerides odorata, Bulbophyllum lilacinum, Cymbidium aloifolium, Dendrobium aphyllum, Luisia zeylanica, Vanda teres, Pholidota imbricata and Rhynchostylis retusa. One threatened Gymnosperm Gnetum oblongum, is also observed in the area. The most common planted species observed in the area are Acacia auriculiformis, Acacia mangium, Tectona grandis, Gmelina arborea, Syzygium grandis, Terminalia arjuna, Aquilaria agallocha and Dipterocarpus turbinatus. The common bamboo species are Bambusa tulda and Melocanna baccifera. Among rattans Calamus erectus and $C$. longisetus are found in the forest. In the homestead gardens, common trees are Artocarpus heterophyllus, Mangifera indica, Areca catechu, Borassus flabellifer, Carica papaya, Citrus grandis, Cocos nucifera and Psidium guajava. 
Table 1. List of species recorded in Teknaf wildlife sanctuary (* means cultivated).

\begin{tabular}{|c|c|c|c|}
\hline Scientific name & Family & Bangla name & Habit \\
\hline Abelmoschus moschatus Medic. & Malvaceae & Mushak dana & Herb \\
\hline Abrus precatorius L. & Fabaceae & Ratti & Climber \\
\hline *Acacia auriculiformis A. Cunn. ex Benth. \& Hook. & Mimosaceae & Akashmoni & Tree \\
\hline A. concinna (Willd.) DC. & Mimosaceae & Banrita & Climber \\
\hline *A. mangium Willd. & Mimosaceae & Belgium & Tree \\
\hline Acanthus ilicifolius L. & Acanthaceae & Hergoza & Shrub \\
\hline Achyranthes aspera L. & Amaranthaceae & Apang & Herb \\
\hline Actephila excelsa (Dalz.) Muell.-Arg. & Euphorbiaceae & - & Shrub \\
\hline Adenia trilobata (Roxb.) Engl. & Passifloraceae & Akandphul & Climber \\
\hline Adina cordifolia Hook. f. ex Brandis & Rubiaceae & Dakrum & Tree \\
\hline Aegialitis rotundifolia Roxb. & Plumbaginaceae & Nuinna & Shrub \\
\hline Aegiceras corniculata (L.) Blanco & Primulaceae & Khoilsha & Shrub \\
\hline *Aegle marmelos (L.) Corr. & Rutaceae & Bel & Tree \\
\hline Aerides multiflora Roxb. & Orchidaceae & - & Epiphyte \\
\hline A. odorata Lour. & Orchidaceae & - & Epiphyte \\
\hline Aerua monsonia Mart. & Amaranthaceae & - & Herb \\
\hline A. sanguinolenta (L.) Blume & Amaranthaceae & - & Herb \\
\hline Ageratum conyzoides L. & Asteraceae & Fulkuri & Herb \\
\hline Aglaonema hookerianum Schott & Araceae & - & Herb \\
\hline Albizia lucidior (Steud.) Nielsen & Mimosaceae & Sil-koroi & Tree \\
\hline A. procera (Roxb.) Benth. & Mimosaceae & Silkoroi & Tree \\
\hline Allophylus cobbe (L.) Raeuschel & Sapindaceae & Chita & Shrub \\
\hline Alocasia acuminata Schott & Araceae & - & Herb \\
\hline A. macrorrhizos (L.) G. Don & Araceae & Mankachu & Herb \\
\hline Alpinia malaccensis (Burm. f.) Rosc. & Zingiberaceae & Deotara & Shrub \\
\hline Alstonia scholaris L. & Apocynaceae & Chatim & Tree \\
\hline Alternanthera philoxeroides (Mart.) Griseb. & Amaranthaceae & Helencha & Herb \\
\hline A. sessilis (L.) R. Br. ex Roem \& Schult. & Amaranthaceae & Upathlenga & Herb \\
\hline Amaranthus gangeticus L. & Amaranthaceae & Shadamayishk & Herb \\
\hline A. spinosus L. & Amaranthaceae & Kanta-nutia & Herb \\
\hline A. viridis $\mathrm{L}$. & Amaranthaceae & Notey Sak & Herb \\
\hline Ammannia multiflora Roxb. & Lythraceae & - & Herb \\
\hline Amomum aromaticum Roxb. & Zingiberaceae & Tara & Shrub \\
\hline Amorphophallus bulbifer (Roxb.) Blume & Araceae & Oll & Herb \\
\hline *Anacardium occidentale L. & Anacardiaceae & Kaju badam & Tree \\
\hline Anisomeles heyneana Wall. ex Benth. & Lamiaceae & - & Herb \\
\hline A. indica (L.) O. Kuntze & Lamiaceae & Gobura & Herb \\
\hline Anisoptera scaphula (Roxb.) Pierre & Dipterocarpaceae & Boilum & Tree \\
\hline *Annona reticulata $\mathrm{L}$. & Annonaceae & Ata & Tree \\
\hline Anodendron paniculatum (Roxb.) A. DC. & Apocynaceae & - & Climber \\
\hline Anogeissus acuminata (Roxb. ex DC.) Guill. \& Perr. & Combretaceae & Chakua & Tree \\
\hline Antidesma acuminatum Wall. & Euphorbiaceae & Chukka & Shrub \\
\hline
\end{tabular}


Table 1 contd.

\begin{tabular}{|c|c|c|c|}
\hline Scientific name & Family & Bangla name & Habit \\
\hline A. ghaesembilla Gaertn. & Euphorbiaceae & Khudijam & Shrub \\
\hline A. roxburghii Wall. ex Tulasne & Euphorbiaceae & - & Shrub \\
\hline Aphanamixis polystachya (Wall.) R. N. Parker & Meliaceae & Pitraj & Tree \\
\hline Aporosa dioica (Roxb.) Muell.-Arg. & Euphorbiaceae & Patakharolla & Tree \\
\hline *Aquilaria agallocha Roxb. & Thymeliaceae & Agar & Tree \\
\hline Ardisia elliptica Thunb. & Myrsinaceae & - & Shrub \\
\hline A. paniculata Roxb. & Myrsinaceae & - & Shrub \\
\hline A. solanacea (Poir.) Roxb. & Myrsinaceae & - & Shrub \\
\hline *Areca catechu L. & Arecaceae & Supari & Tree \\
\hline Argyreia capitiformis (Poir.) van Cheek Oostr. & Convolvulaceae & - & Climber \\
\hline A. roxburghii Choisy & Convolvulaceae & - & Climber \\
\hline Aristolochia tagala Cham. & Aristolochiaceae & Ishwarmul & Climber \\
\hline Artocarpus chaplasha Roxb. & Moraceae & Chapalish & Tree \\
\hline *A. heterophyllus Lamk. & Moraceae & Kanthal & Tree \\
\hline Arundo donax L. & Poaceae & Nal & Herb \\
\hline *Averrhoa bilimbi L. & Oxalidaceae & Bilimbi & Tree \\
\hline *A. carambola L. & Oxalidaceae & Kamranga & Tree \\
\hline Avicennia alba Blume & Verbenaceae & Sada baen & Tree \\
\hline A. marima (Forssk.) Vierh. & Verbenaceae & Moricha baen & Tree \\
\hline A. officinalis L. & Verbenaceae & Kala baen & Tree \\
\hline Axonopus compressus (Sw.) P. Beauv. & Poaceae & Dhakagash & Herb \\
\hline *Azadirachta indica A. Juss. & Meliaceae & Neem & Tree \\
\hline Bacopa monieri (L.) Pennell & Scrophulariaceae & Brammi & Herb \\
\hline Bambusa balcooa Roxb. & Poaceae & barak bash & Tree \\
\hline B. polymorpha Munro & Poaceae & Parua bash & Tree \\
\hline B. tulda Roxb. & Poaceae & Mitinga bash & Tree \\
\hline Barringtonia acutangula (L.) Gaertn. & Lecythidaceae & Hizol & Tree \\
\hline Bauhinia acuminata L. & Caesalpiniaceae & Kanson & Shrub \\
\hline Begonia roxburghii (Miq.) DC. & Begoniaceae & - & Herb \\
\hline Blumea lacera (Burm. f.) DC. & Asteraceae & Kukurmuta & Herb \\
\hline B. membranacea Wall. ex DC. & Asteraceae & Shialmutra & Herb \\
\hline B. virens Wall. ex DC. & Asteraceae & - & Herb \\
\hline Bombax ceiba L. & Bombacaceae & Shimul & Tree \\
\hline B. insigne Wall. & Bombacaceae & Bonshimul & Tree \\
\hline *Borassus flabellifer L. & Arecaceae & Tal & Tree \\
\hline Borreria articularis (L. f.) Williams & Rubiaceae & Antharogia & Herb \\
\hline B. latifolia (Aublet) K. Schum. & Rubiaceae & Ghuiojhill sak & Herb \\
\hline Breynia retusa (Dennst.) Alston & Euphorbiaceae & Silpati & Shrub \\
\hline B. vitis-idaea (Burm. f.) C. E. C. Fischer & Euphorbiaceae & - & Shrub \\
\hline Bridelia retusa (L.) A. Juss. & Euphorbiaceae & Kata koi & Shrub \\
\hline B. stipularis (L.) Blume & Euphorbiaceae & Pat khowi & Climber \\
\hline Brownlowia elata Roxb. & Tiliaceae & Massjot & Tree \\
\hline
\end{tabular}


Table 1 contd.

\begin{tabular}{|c|c|c|c|}
\hline Scientific name & Family & Bangla name & Habit \\
\hline Bruguiera gymnorrhiza (L.) Lamk. & Rhizophoraceae & Goran & Tree \\
\hline Bulbophyllum lilacinum Ridl. & Orchidaceae & Parchallow & Epiphyte \\
\hline Butea monosperma (Lamk.) Taub. & Fabaceae & Polash & Tree \\
\hline Byttneria pilosa Roxb. & Sterculiaceae & Harbanga lata & Climber \\
\hline Caesalpinia bonduc (L.) Roxb. & Caesalpiniaceae & Nata & Climber \\
\hline C. crista L. & Caesalpiniaceae & Letkanta & Climber \\
\hline${ }^{*}$ C. pulcherrima (L.) Swartz & Caesalpiniaceae & Radhachura & Tree \\
\hline *Cajanus cajan (L.) Millsp. & Fabaceae & Orhor & Shrub \\
\hline Calamus erectus Roxb. & Arecaceae & Kadam bet & Shrub \\
\hline C. longisetus Griff. & Arecaceae & Udombet & Climber \\
\hline Calliandra umbrosa (Wall.) Benth. & Mimosaceae & Chotto betmar & Shrub \\
\hline Callicarpa arborea Roxb. & Verbenaceae & Bormala & Tree \\
\hline C. macrophylla Vahl & Verbenaceae & Bormala & Tree \\
\hline Calophyllum polyanthum Wall. ex Choisy & Clusiaceae & Keroli & Tree \\
\hline Calotropis procera (Ait.) R. Br. & Asclepiadaceae & Akand & Shrub \\
\hline Calycopteris floribunda (Roxb.) Lamk. & Combretaceae & Guicha lata & Climber \\
\hline Campanumoea lancifolia (Roxb.) Merr. & Campanulaceae & - & Herb \\
\hline Carex indica $\mathrm{L}$. & Сyperaceae & - & Herb \\
\hline *Carica papaya L. & Caricaceae & Pepe & Tree \\
\hline Cassia fistula $\mathrm{L}$. & Caesalpiniaceae & Sonalu & Tree \\
\hline Castanopsis tribuloides (Smith) A. DC. & Fagaceae & Hingra & Tree \\
\hline Casuarina equisetifolia Forst. & Casuarinaceae & Jau & Tree \\
\hline Cayratia japonica (Thunb.) Gagnep. & Vitaceae & - & Climber \\
\hline Ceiba pentandra (L.) Gaertn. & Bombacaceae & Tula & Tree \\
\hline Celtis timorensis Span. & Ulmaceae & Datarchua & Shrub \\
\hline Centella asiatica (L.) Urban & Apiaceae & Thaimonshak & Herb \\
\hline Ceriops decandra (Griff.) Ding Hou & Rhizophoraceae & Khemo & Tree \\
\hline Chromolaena odorata (L.) King \& Robinson & Asteraceae & Assamlata & Shrub \\
\hline Chrysopogon aciculatus (Retz.) Trin. & Poaceae & Premkanta & Herb \\
\hline Chukrasia tabularis A. Juss. & Meliaceae & Chikrasi & Tree \\
\hline Cinnamomum iners Reinw. ex Blume & Lauraceae & Tejmul & Tree \\
\hline Cissampelos pareira $\mathrm{L}$. & Menispermaceae & Tubaki-lata & Climber \\
\hline Cissus adnata Roxb. & Vitaceae & Aliangalata & Climber \\
\hline *Citrus aurantium L. & Rutaceae & Komala & Shrub \\
\hline${ }^{*}$ C. grandis (L.) Osbeck & Rutaceae & Jambura & Tree \\
\hline Clausena heptaphylla (Roxb.) Wight \& Arn. ex Steud. & Rutaceae & Ponkarpur & Shrub \\
\hline C. suffruticosa (Roxb.) Wight \& Arn. & Rutaceae & Panbilash & Shrub \\
\hline Cleome rutidosperma DC. & Capparaceae & - & Herb \\
\hline C. viscosa L. & Capparaceae & Hurhuria & Herb \\
\hline Clerodendrum inerme (L.) Gaertn. & Verbenaceae & Bamjui & Shrub \\
\hline C. viscosum Vent. & Verbenaceae & Bhant & Shrub \\
\hline Clitoria ternatea $\mathrm{L}$. & Fabaceae & Aparjita & Climber \\
\hline
\end{tabular}


Table 1 contd.

\begin{tabular}{|c|c|c|c|}
\hline Scientific name & Family & Bangla name & Habit \\
\hline Cnesmone javanica Blume & Euphorbiaceae & Chutra & Climber \\
\hline *Cocos nucifera L. & Arecaceae & Narikel & Tree \\
\hline Colocasia esculenta (L.) Schott & Araceae & Kachu & Herb \\
\hline C. heterochroma H. Li et Z.X. Wei & Araceae & - & Herb \\
\hline C. oresbia A. Hay & Araceae & - & Herb \\
\hline Combretum decandrum Roxb. & Combretaceae & Sada guicha & Climber \\
\hline Commelina benghalensis L. & Commelinaceae & Kanchira & Herb \\
\hline C. erecta L. & Commelinaceae & Jata kanchira & Herb \\
\hline C. longifolia Lamk. & Commelinaceae & Pani kanchira & Herb \\
\hline Costus speciosus (Koenig ex Retz.) Smith & Costaceae & Keumul & Herb \\
\hline Crateva magna (Lour.) DC. & Capparaceae & Borun & Tree \\
\hline Crinum amoenum Roxb. & Liliaceae & Bopiaz & Herb \\
\hline C. asiaticum L. & Liliaceae & Gor-rosun & Herb \\
\hline Crotalaria juncea L. & Fabaceae & Junjuni & Herb \\
\hline C. pallida Ait. & Fabaceae & Jhunjhni & Herb \\
\hline Curculigo orchioides Gaertn. & Liliaceae & Talmuli & Herb \\
\hline C. recurvata Dryand. & Liliaceae & Satipata & Herb \\
\hline Curcuma amada Roxb. & Zingiberaceae & Shadi & Herb \\
\hline C. latifolia Rosc. & Zingiberaceae & Amada & Herb \\
\hline C. zedoaria (Christm.) Rosc. & Zingiberaceae & Shoti & Herb \\
\hline Cuscuta reflexa Roxb. & Cuscutaceae & Shornalata & Climber \\
\hline Cyclea barbata Miers & Menispermaceae & Patalpur & Climber \\
\hline Cymbidium aloifolium (L.) Sw. & Orchidaceae & Churi & Epiphyte \\
\hline Cynodon dactylon (L.) Pers. & Poaceae & Durba & Herb \\
\hline Cyperus cyperoides (L.) O. Ktze. & Cyperaceae & - & Herb \\
\hline C. iria L. & Cyperaceae & - & Herb \\
\hline C. kyllingia Endl. & Cyperaceae & - & Herb \\
\hline C. laxus Lamk. var. laxus & Cyperaceae & - & Herb \\
\hline C. pilosus Vahl & Cyperaceae & - & Herb \\
\hline C. rotundus $\mathrm{L}$. & Cyperaceae & - & Herb \\
\hline Dalbergia rimosa Roxb. & Fabaceae & - & Shrub \\
\hline D. sissoo Roxb. & Fabaceae & - & Tree \\
\hline D. spinosa Roxb. & Fabaceae & - & Shrub \\
\hline D. stipulacea Roxb. & Fabaceae & Dadbari & Climber \\
\hline D. tamarindifolia Roxb. & Fabaceae & - & Shrub \\
\hline D. volubilis Roxb. & Fabaceae & Ankilata & Shrub \\
\hline Datura metel L. & Solanaceae & Dhatura & Shrub \\
\hline Dehaasia kurzii King ex Hook. f. & Lauraceae & Modonmosta & Tree \\
\hline *Delonix regia Rafin. & Caesalpiniaceae & krishnachura & Tree \\
\hline Dendrobium aphyllum (Roxb.) Fischer & Orchidaceae & - & Epiphyte \\
\hline Derris scandens (Roxb.) Benth. & Fabaceae & Kalilata & Climber \\
\hline D. trifoliata Lour. & Fabaceae & Melata & - \\
\hline
\end{tabular}


Table 1 contd.

\begin{tabular}{|c|c|c|c|}
\hline Scientific name & Family & Bangla name & Habit \\
\hline Desmodium heterocarpon (L.) DC. & Fabaceae & - & Herb \\
\hline D. heterophyllum (Willd.) DC. & Fabaceae & - & Herb \\
\hline D. pulchellum (L.) Benth. & Fabaceae & Juta salpani & Shrub \\
\hline D. styracifolium (Osb.) Merr. & Fabaceae & - & Herb \\
\hline D. triflorum (L.) DC. & Fabaceae & Kulalia & Herb \\
\hline D. triquetrum (L.) DC. & Fabaceae & - & Herb \\
\hline D. triquetrum (L.) DC. subsp. alatum (DC.) Prain & Fabaceae & - & Herb \\
\hline Dichopsis polyantha Benth. & Sapotaceae & Tali & Tree \\
\hline Digitaria sanguinalis (L.) Scop. & Poaceae & Makunjill & Herb \\
\hline D. violascens Link & Poaceae & - & Herb \\
\hline Dillenia indica L. & Dilleniaceae & Chalta & Tree \\
\hline D. pentagyna Roxb. & Dilleniaceae & Hargenza & Tree \\
\hline D. scabrella Roxb. ex Wall. & Dilleniaceae & Ekuish & Tree \\
\hline Dioscorea alata L. & Dioscoreaceae & Suprialu & Climber \\
\hline D. belophylla (Prain) Voigt ex Haines & Dioscoreaceae & - & Climber \\
\hline D. bulbifera L. var. bulbifera L. & Dioscoreaceae & Ratal, Bon alu & Climber \\
\hline D. kamoonensis Kunth & Dioscoreaceae & - & Climber \\
\hline D. melanophyma Prain \& Burkill & Dioscoreaceae & - & Climber \\
\hline D. oppositifolia L. & Dioscoreaceae & Randrealeku & Climber \\
\hline D. pentaphylla L. & Dioscoreaceae & - & Climber \\
\hline Di. trinerva Roxb. & Dioscoreaceae & - & Climber \\
\hline Dipterocarpus alatus Roxb. ex G. Don & Dipterocarpaceae & Dholi garjan & Tree \\
\hline D. costatus Gaertn. & Dipterocarpaceae & Sil garjan & Tree \\
\hline D. gracilis Blume & Dipterocarpaceae & - & Tree \\
\hline D. turbinatus Gaertn. & Dipterocarpaceae & Kaligarjan & Tree \\
\hline Dracaena spicata Roxb. & Agavaceae & Dracaena & Shrub \\
\hline Duranta repens L. & Verbenaceae & Katamehedi & Shrub \\
\hline Dysolobium dolichoides (Roxb.) Prain & Fabaceae & - & Climber \\
\hline Echinochloa colonum (L.) Link & Poaceae & Shama grass & Herb \\
\hline Eclipta alba (L.) Hassk. & Asteraceae & Keshoraj & Herb \\
\hline Eichhornia crassipes (Mart.) Solms & Pontederiaceae & Kachuripana & Herb \\
\hline Elaeocarpus floribundus Blume & Elaeocarpaceae & Belphoi & Tree \\
\hline E. robustus Roxb. & Elaeocarpaceae & Jalpai & Tree \\
\hline Elatostema sesquifolium (Blume) Hassk. & Urticaceae & - & Herb \\
\hline Eleocharis palustris (L.) R. Br. & Cyperaceae & - & Herb \\
\hline Elephantopus scaber L. & Asteraceae & - & Herb \\
\hline Eleusine indica (L.) Gaertn. & Poaceae & Malan kuri & Herb \\
\hline Endospermum chinense Benth. & Euphorbiaceae & - & Tree \\
\hline Engelhardtia spicata Lesch. ex Blume & Guglandaceae & Zalna & Tree \\
\hline Enhydra fluctuans Lour. & Asteraceae & Helencha & Herb \\
\hline Entada scandens auct. non Benth. & Mimosaceae & Gila & Climber \\
\hline Eragrostis tenella (L.) P. Beauv. ex Roem. \& Schult. & Poaceae & Koni grass & Herb \\
\hline
\end{tabular}


Table 1 contd.

\begin{tabular}{|c|c|c|c|}
\hline Scientific name & Family & Bangla name & Habit \\
\hline Eranthemum strictum Coleb. ex Roxb. & Acanthaceae & - & Herb \\
\hline *Eryngium foetidum L. & Apiaceae & Katkatriabaho & Herb \\
\hline Erythrina fusca Lour. & Fabaceae & Mandar & Tree \\
\hline E. indica Lamk. & Fabaceae & Mandar & Tree \\
\hline E. ovalifolia Roxb. & Fabaceae & Mandar & Tree \\
\hline Etlingera linguiformis (Roxb.) R. M. Smith & Zingiberaceae & - & Shrub \\
\hline Euphorbia hirta L. & Euphorbiaceae & Dudhia & Herb \\
\hline E. thymifolia L. & Euphorbiaceae & Dudhiya & Herb \\
\hline Evolvulus nummularius (L.) L. & Convolvulaceae & - & Herb \\
\hline Excoecaria agallocha $\mathrm{L}$. & Euphorbiaceae & Gewa & Tree \\
\hline Ficus altissima Blume & Moraceae & Bot & Tree \\
\hline F. benghalensis L. & Moraceae & Bot & Tree \\
\hline F. benjamina L. & Moraceae & Jir & Tree \\
\hline F. fistulosa Reinw. ex Blume & Moraceae & - & Shrub \\
\hline F. hispida L. f. & Moraceae & Dumur & Herb \\
\hline F. pumila L. & Moraceae & Dewall dumar & Climber \\
\hline F. racemosa L. & Moraceae & Jagya dumar & Tree \\
\hline F. rumphii Blume & Moraceae & - & Tree \\
\hline F. scandens Buch.-Ham. & Moraceae & - & Climber \\
\hline F. semicordata Buch.-Ham. ex Smith & Moraceae & Chotochorkigu & Tree \\
\hline F. virens Ait. & Moraceae & Pakur & Tree \\
\hline Fimbristylis dichotoma (L.) Vahl subsp. dichotoma & Cyperaceae & - & Herb \\
\hline F. miliacea (L.) Vahl & Cyperaceae & - & Herb \\
\hline Fissistigma polyanthum (Hook. f. \& Thom.) Merr. & Annonaceae & - & Climber \\
\hline Flacourtia indica (Burm. f.) Merr. & Flacourtiaceae & Paniala & Shrub \\
\hline F. inermis Roxb. & Flacourtiaceae & - & Shrub \\
\hline Flagellaria indica L. & Flagellariacea & - & Climber \\
\hline Flemingia macrophylla (Willd.) O. Kuntze ex Merr. & Fabaceae & Bara shaphan & Shrub \\
\hline F. strobilifera (L.) R. Br. & Fabaceae & - & Shrub \\
\hline Floscopa scandens Lour. & Commelinaceae & - & Herb \\
\hline Garcinia cowa Roxb. ex DC. & Clusiaceae & Kau phal & Tree \\
\hline G. xanthochymus Hook. f. ex T. Anders. & Clusiaceae & Dayphal & Tree \\
\hline $\begin{array}{l}\text { Garuga floribunda Decne. var. gamblei (King ex } \\
\text { Smith) Kalkman }\end{array}$ & Burseraceae & Jongli jiga & Tree \\
\hline G. pinnata Roxb. & Burseraceae & Jeolbhadi & Tree \\
\hline Geissapsis cristata Wight \& Arn. & Fabaceae & - & Herb \\
\hline Geodorum densiflorum (Lamk.) Schltr. & Orchidaceae & - & Herb \\
\hline Globba multiflora Wall. ex Baker & Zingiberaceae & - & Herb \\
\hline Gloriosa superba L. & Liliaceae & Ulatchandal & Climber \\
\hline Glycosmis mauritiana (Lamk.) Tanaka & Rutaceae & - & Shrub \\
\hline G. pentaphylla (Retz.) A. DC. & Rutaceae & Datmajan & Shrub \\
\hline Gmelina arborea Roxb. & Verbenaceae & Gamari & Tree \\
\hline
\end{tabular}


Table 1 ontd.

\begin{tabular}{|c|c|c|c|}
\hline Scientific name & Family & Bangla name & Habit \\
\hline Gnetum oblongum L. & Gnetaceae & - & Climber \\
\hline Goniothalamus sesquipedalis (Wall.) Hook. F. \& Thom. & Annonaceae & - & Shrub \\
\hline Gouania tiliaefolia Lamk. & Rhamnaceae & - & Climber \\
\hline Grewia microcos L. & Tiliaceae & Assar & Shrub \\
\hline Gymnopetalum cochinchinense (Lour.) Kurz & Cucurbitaceae & - & Climber \\
\hline Gynostemma pentaphylla (Thumb.) Makino. & Vitaceae & - & Climber \\
\hline Hedyotis scandens Roxb. & Rubiaceae & Bish lata & Herb \\
\hline Heliotropium indicum L. & Boraginaceae & Hatisun & Herb \\
\hline Hemarthria protensa Steud. & Poaceae & Chalia & Herb \\
\hline Hemidesmus indicus (L.) R. Br. & Asclepiadaceae & Anantamul & Climber \\
\hline Hemigraphis hirta (Vahl) T. Anders. & Acanthaceae & - & Herb \\
\hline Heritiera fomes Buch.-Ham. & Sterculiaceae & Sundari & Tree \\
\hline Heterophragma adenophylla (Wall. ex G. Don) Benth. & Bignoniaceae & Dakrum & Tree \\
\hline *Hibiscus rosa-sinensis L. & Malvaceae & Joba & Shrub \\
\hline H. tiliaceus L. & Malvaceae & Bolla & Shrub \\
\hline Holarrhena antidysenterica (L.) Wall. ex Decne. & Apocynaceae & Kurchi & Shrub \\
\hline Holigarna longifolia Roxb. & Anacardiaceae & Barala & Tree \\
\hline Homalomena aromatica (Roxb. ex Sim) Schott & Araceae & - & Herb \\
\hline Hopea odorata Roxb. & Dipterocarpaceae & Telsur & Tree \\
\hline Hoya parasitica (Roxb.) Wall. ex Wight & Asclepiadaceae & Pargacha & Climber \\
\hline Hydrolea zeylanica (L.) Vahl & Hydrophyllaceae & - & Herb \\
\hline Hygrophila polysperma (Roxb.) T. Anders. & Acanthaceae & - & Herb \\
\hline Hymenodictyon excelsum (Roxb.) Wall. & Rubiaceae & Bhuikadam & Tree \\
\hline Hyptis brevipes Poit. & Lamiaceae & - & Herb \\
\hline H. suaveolens (L.) Poit. & Lamiaceae & Tokma & Herb \\
\hline Ichnocarpus frutescens (L.) R. Br. & Apocynaceae & Shamalata & Climber \\
\hline $\begin{array}{l}\text { Imperata cylindrica (L.) P. Beauv. var. latifolia (Hook. } \\
\text { f.) C. E. Hubb. }\end{array}$ & Poaceae & Ulu & Herb \\
\hline Ipomoea aquatica Forssk. & Convolvulaceae & Kalmi sak & Climber \\
\hline I. fistulosa Mart. ex Choisy & Convolvulaceae & Dholkalmi & Shrub \\
\hline I. mauritiana Jacq. & Convolvulaceae & Huffta alu & Climber \\
\hline I. pes-caprae (L.) R. Br. & Convolvulaceae & Chagalkhuri & Climber \\
\hline I. quamoclit L. & Convolvulaceae & Gate phul & Climber \\
\hline Ischaemum indicum (Houtt.) Merr. & Poaceae & Toto grass & Herb \\
\hline Ixora acuminata Roxb. & Rubiaceae & - & Shrub \\
\hline I. javanica DC. & Rubiaceae & Rangan & Shrub \\
\hline I. pavetta Andr. & Rubiaceae & Swet rangan & Shrub \\
\hline *Jasminum auriculatum Vahl & Oleaceae & Jui & Climber \\
\hline J. grandiflorum L. & Oleaceae & Wild jasmin & Climber \\
\hline J. scandens Vahl & Oleaceae & - & Shrub \\
\hline Jatropha curcas L. & Euphorbiaceae & Sadajeol & Tree \\
\hline Justicia gendarussa Burm. f. & Acanthaceae & Nilnishinda & Shrub \\
\hline
\end{tabular}


Table 1 contd.

\begin{tabular}{|c|c|c|c|}
\hline Scientific name & Family & Bangla name & Habit \\
\hline J. simplex D. Don. & Acanthaceae & Jogathmardan & Shrub \\
\hline Kaempferia galanga L. & Zingiberaceae & Tiutara & Herb \\
\hline *Lagenaria siceraria (Molina) Standl. & Cucurbitaceae & Lau & Herb \\
\hline Lagerstroemia speciosa (L.) Pers. & Lythraceae & Jarul & Tree \\
\hline Lannea coromandelica (Houtt.) Merr. & Anacardiaceae & Jiga & Tree \\
\hline Lantana camara L. & Verbenaceae & Lantana & Shrub \\
\hline Laportea interrupta (L.) Chew & Urticaceae & Lal Bichuti & Herb \\
\hline Lasia spinosa (L.) Thw. & Araceae & Kantakachu & Herb \\
\hline *Lawsonia inermis L. & Lythraceae & Mehedi & Shrub \\
\hline Leea acuminata Wall. & Leeaceae & Phupharia & Shrub \\
\hline L. aequata $\mathrm{L}$. & Leeaceae & - & Shrub \\
\hline L. crispa L. & Leeaceae & Banchilata & Shrub \\
\hline L. indica Merr. & Leeaceae & - & Shrub \\
\hline Lepidagathis incurva Buch.-Ham. ex D. Don & Acanthaceae & - & Herb \\
\hline Lepisanthes rubiginosa (Roxb.) Leenh. & Sapindaceae & Baraharina & Shrub \\
\hline *Leucaena leucocephala (Lamk.) de Wit. & Mimosaceae & Ipli-ipil & Tree \\
\hline Leucas aspera (Willd.) Link & Lamiaceae & Dandakalash & Herb \\
\hline L. lavandulaefolia Smith & Lamiaceae & Gaochia & Herb \\
\hline Limnophila indica (L.) Druce & Scrophulariaceae & Pani karpur & Herb \\
\hline Limonia acidissima $\mathrm{L}$. & Rutaceae & Koethbel & Tree \\
\hline $\begin{array}{l}\text { Lithocarpus elegans var. elegans (Blume) Hatus. ex } \\
\text { Soepad. }\end{array}$ & Fagaceae & Barabatna & Tree \\
\hline Litsea glutinosa (Lour.) Robinson & Lauraceae & Menda & Tree \\
\hline L. monopetala (Roxb.) Pers. & Lauraceae & Kukuchita & Tree \\
\hline Lophopetalum wightianum Arn. & Celastraceae & Rokton & Tree \\
\hline Ludwigia adscendens (L.) Hara & Onagraceae & Mulsi & Herb \\
\hline L. hyssopifolia (G. Don) Exell apud A. \& R. Fernandes & Onagraceae & - & Herb \\
\hline Luffa graveolens Roxb. & Cucurbitaceae & Pahari dhundul & Climber \\
\hline Luisia zeylanica Lindl. & Orchidaceae & - & Epiphyte \\
\hline Macaranga peltata (Roxb.) Muell.-Arg. & Euphorbiaceae & Bura & Shrub \\
\hline Macrosolen cochinchinensis (Lour.) Van Tiegh. & Loranthaceae & Porgasa & Parasite \\
\hline Maesa indica (Roxb.) A. DC. & Myrsinaceae & Ramjoni & Shrub \\
\hline M. ramentacea (Roxb.) A. DC. & Myrsinaceae & Maricha & Shrub \\
\hline Mallotus philippensis (Lamk.) Muell.-Arg. & Euphorbiaceae & Kamela & Shrub \\
\hline Mangifera indica L. & Anacardiaceae & Aam & Tree \\
\hline M. sylvatica Roxb. & Anacardiaceae & Uriam & Tree \\
\hline Manihot esculenta Crantz & Euphorbiaceae & Kasava & Shrub \\
\hline Manilkara hexandra (Roxb.) Dubard & Sapotaceae & Khirni & Shrub \\
\hline Mantisia radicalis (Roxb.) D. P. Dam \& N. Dam & Zingiberaceae & - & Herb \\
\hline M. spathulata Schult. & Zingiberaceae & - & Herb \\
\hline Maranta arundinacea L. & Marantaceae & Ararot & Herb \\
\hline Melastoma malabathricum L. & Melastomaceae & Futki & Shrub \\
\hline
\end{tabular}


Table 1 contd.

\begin{tabular}{|c|c|c|c|}
\hline Scientific name & Family & Bangla name & Habit \\
\hline *Melia azedarach L. & Meliaceae & Ghura neem & Tree \\
\hline Melocanna baccifera (Roxb.) Kurz & Poaceae & Moli bash & Tree \\
\hline Melochia corchorifolia L. & Sterculiaceae & Tiki okra & Herb \\
\hline M. umbellata (L.) Hallier f. & Convolvulaceae & Sadakalmi & Climber \\
\hline Michelia champaca L. & Magnoliaceae & Champa & Tree \\
\hline Micromelum minutum (G. Forster) Wight \& Arn. & Rutaceae & Koroiphula & Shrub \\
\hline Mikania cordata (Burm. f.) Robinson & Asteraceae & Assamlata & Climber \\
\hline Miliusa globosa (DC.) G. Panigr. \& Mishra & Annonaceae & Tasbi & Climber \\
\hline Millettia cinerea Benth. & Fabaceae & - & Herb \\
\hline Mimosa invisa Mart. ex Colla. & Mimosaceae & Bara lajjabati & Herb \\
\hline M. pudica L. & Mimosaceae & Lajjabati & Herb \\
\hline Mitragyna rotundifolia (Roxb.) O. Kuntze & Rubiaceae & Rang kat & Tree \\
\hline Molineria recurvata (Dryand.) Herbert. & Liliaceae & - & Herb \\
\hline Mollugo pentaphylla L. & Moraceae & Khetpapra & Herb \\
\hline Momordica dioica Roxb. ex Willd. & Cucurbitaceae & Bonkorolla & Climber \\
\hline Monochoria vaginalis (Burm. f.) Presl & Pontederiaceae & Nukha & Herb \\
\hline Mucuna pruriens (L.) DC. & Fabaceae & Alkushi & Climber \\
\hline Murraya koenigii (L.) Spreng. & Rutaceae & Kamini & Tree \\
\hline Musa acuminata Colla & Musaceae & - & Herb \\
\hline Mussaenda frondosa $\mathrm{L}$. & Rubiaceae & Kalasonia & Shrub \\
\hline M. roxburghii Hook. f. & Rubiaceae & Silchuri & Shrub \\
\hline Nelsonia canescens (Lamk.) Spreng. & Acanthaceae & Parmul & Herb \\
\hline Neolamarckia cadamba (Roxb.) Bosser & Rubiaceae & Kadam & Tree \\
\hline Nymphaea rubra Roxb. ex Andr. & Nympheaceae & Lal Shaphla & Herb \\
\hline Nypa fruticans Wurmb. & Arecaceae & Golpata & Shrub \\
\hline Ocimum americanum $\mathrm{L}$. & Lamiaceae & Tulsi & Herb \\
\hline *O. gratissimum L. & Lamiaceae & Ramtulsi & Herb \\
\hline Oplismenus burmanii (Retz.) P. Beauv. & Poaceae & - & Herb \\
\hline Opuntia dillenii Haw. & Cactaceae & Phanimansa & Herb \\
\hline Oreocnide integrifolia (Gaud.) Miq. & Urticaceae & Horhuta & Shrub \\
\hline Ormosia robusta (Roxb.) Baker & Fabaceae & - & Tree \\
\hline Oroxylum indicum (L.) Kurz & Bignoniaceae & Thona & Tree \\
\hline Osbeckia aspericaulis Hook. f. ex Triana & Melastomaceae & - & Shrub \\
\hline Oxyceros kunstleri (King \& Gamble) Tirveng. & Rubiaceae & Moishkanta & Climber \\
\hline Pandanus foetidus Roxb. & Pandanaceae & Keyakanta & Shrub \\
\hline P. odorus Ridl. & Pandanaceae & Keyakanta & Shrub \\
\hline Panicum brevifolium L. & Poaceae & - & Herb \\
\hline P. notatum Retz. & Poaceae & - & Herb \\
\hline Papilionanthe teres (Roxb.) Schltr. & Orchidaceae & Vanda & Epiphyte \\
\hline Paspalum scrobiculatum L. & Poaceae & Kodoa phan & Herb \\
\hline Passiflora foetida L. & Passifloraceae & Jhumku lata & Climber \\
\hline Peliosanthes teta Andr. & Haemodoraceae & Napi gach & Herb \\
\hline
\end{tabular}


Table 1 contd.

\begin{tabular}{|c|c|c|c|}
\hline Scientific name & Family & Bangla name & Habit \\
\hline Pentatropis capensis (L. f.) Bullock & Asclepiadaceae & - & Climber \\
\hline Persicaria flaccida (Meissn.) H. Gross ex Loesen. & Polygonaceae & Lal-bishkatali & Herb \\
\hline P. hydropiper (L.) Spach & Polygonaceae & Lal-kukri & Herb \\
\hline P. orientalis (L.) Spach & Polygonaceae & Bara panimorich & Herb \\
\hline Phaulopsis imbricata (Forssk.) Sweet & Acanthaceae & - & Herb \\
\hline Phoebe lanceolata (Nees) Nees & Lauraceae & - & Shrub \\
\hline Phoenix sylvestris Roxb. & Arecaceae & Khejur & Tree \\
\hline Pholidota imbricata Hook. f. & Orchidaceae & - & Epiphyte \\
\hline Phrynium imbricatum Roxb. & Marantaceae & Pituli pata & Shrub \\
\hline Phyla nodiflora (L.) Greene & Verbenaceae & Bakkumgula & Herb \\
\hline Phyllanthus emblica L. & Euphorbiaceae & Amlaki & Tree \\
\hline P. niruri L. & Euphorbiaceae & Bhuiamla & Tree \\
\hline$P$. reticulatus Poir. & Euphorbiaceae & Chitki & Shrub \\
\hline Physalis minima L. & Solanaceae & Fotka & Herb \\
\hline Pilea melastomoides (Poir.) Wedd. & Urticaceae & - & Shrub \\
\hline Piper betle L. & Piperaceae & Pan & Climber \\
\hline P. sylvaticum Roxb. & Piperaceae & Ban pan & Climber \\
\hline Pithecellobium angulatum Benth. & Mimosaceae & Kurmar & Tree \\
\hline Pogonatherum crinitum (Thunb.) Kunth & Poaceae & - & Herb \\
\hline P. paniceum (Lamk.) Hack. & Poaceae & Choto bush & Herb \\
\hline Polygonum plebeium R. Br. & Polygonaceae & Mechu sak & Herb \\
\hline Pongamia pinnata (L.) Pierre & Fabaceae & - & Tree \\
\hline Pothos scandens L. & Araceae & Batilata & Climber \\
\hline Pouzolzia zeylanica (L.) Benn. & Urticaceae & Kulla kuri & Herb \\
\hline Premna esculenta Roxb. & Verbenaceae & Lallong & Shrub \\
\hline Protium serratum (Wall. ex Coelbr.) EngL. & Burseraceae & Gutgutia & Tree \\
\hline Psidium guajava $\mathrm{L}$. & Myrtaceae & Piara & Shrub \\
\hline Pterospermum semisagittatum Buch.-Ham. ex Roxb. & Sterculiaceae & Ban-assar & Tree \\
\hline Quercus gomeziana A. Camus & Fagaceae & Batna & Tree \\
\hline Randia dumetorum Lamk. & Rubiaceae & Mankanta & Shrub \\
\hline Rhaphidophora grandis Schott & Araceae & - & Climber \\
\hline Rhizophora mucronata Poir. & Rhizophoraceae & - & Tree \\
\hline Rhynchostylis retusa (L.) Blume & Orchidaceae & Foxtail & Epiphyte \\
\hline Rhynchotechum ellipticum (Diet.) DC. & Gesneriaceae & - & Shrub \\
\hline Ricinus communis L. & Euphorbiaceae & Reri & Herb \\
\hline Rotala indica (Willd.) Koehne & Lythraceae & - & Herb \\
\hline R. rotundifolia (Buch.-Ham. ex Roxb.) Koehne & Lythraceae & - & Herb \\
\hline Rungia pectinata (L.) Nees. in Wall. & Acanthaceae & Pindi & Herb \\
\hline Saccharum arundinaceum Retz. & Poaceae & Teng & Herb \\
\hline S. spontaneum L. & Poaceae & Kash & Herb \\
\hline Samanea saman (Jacq.) Merr. & Mimosaceae & Pandi korai & Tree \\
\hline Sambucus canadensis L. & Caprifoliaceae & Hoklati & Tree \\
\hline
\end{tabular}


Table 1 contd.

\begin{tabular}{|c|c|c|c|}
\hline Scientific name & Family & Bangla name & Habit \\
\hline Sapindus saponaria L. & Sapindaceae & Ritha & Tree \\
\hline Saraca thaipingensis Cantley ex Prain & Caesalpiniaceae & Ashok & Shrub \\
\hline Sarcochlamys pulcherrima Gaudich. & Urticaceae & Achila & Shrub \\
\hline Sarcolobus carinatus Wall. & Asclepiadaceae & Baoli lata & Climber \\
\hline Schefflera bengalensis Gamble & Araliaceae & - & Climber \\
\hline Scoparia dulcis L. & Scrophulariaceae & Bandhani & Herb \\
\hline Semicarpus anacardium L.f. & Anacardiaceae & Bhela & Tree \\
\hline Senna alata (L.) Roxb. & Caesalpiniaceae & Dadmordon & Shrub \\
\hline S. obtusifolia (L.) Irwin \& Barneby & Caesalpiniaceae & Chakunda & Herb \\
\hline S. occidentalis Roxb. & Caesalpiniaceae & Eski & Shrub \\
\hline S. sophera (L.) Roxb. & Caesalpiniaceae & Kalkesunde & Herb \\
\hline S. tora (L.) Roxb. & Caesalpiniaceae & Chakunda & Herb \\
\hline Sesbania grandiflora (L.) Poir. & Fabaceae & Bokful & Shrub \\
\hline Setaria glauca (L.) P. Beauv. & Poaceae & Bajra & Herb \\
\hline Shorea robusta Roxb. ex Gaertn. f. & Dipterocarpaceae & Sal & Tree \\
\hline Sida acuta Burm. f. & Malvaceae & Nakphul & Herb \\
\hline S. cordata (Burm. f.) Borss. & Malvaceae & Junka & Herb \\
\hline S. cordifolia L. & Malvaceae & Berela & Herb \\
\hline S. rhombifolia L. & Malvaceae & Lal-berela & Herb \\
\hline Smilax ferox Wall. ex Kunth & Smilacaceae & Kumari lata & Climber \\
\hline S. laurifolia L. & Smilacaceae & Kumari lata & Climber \\
\hline S. ovalifoila Roxb. & Smilacaceae & Kumari lata & Climber \\
\hline S. perfoliata Lour. & Smilacaceae & Kumari lata & Climber \\
\hline Solanum barbisetum Nees & Solanaceae & - & Shrub \\
\hline S. capsicoides All. & Solanaceae & Betbegun & Shrub \\
\hline S. lasiocarpum Dunal & Solanaceae & Beregul & Shrub \\
\hline S. nigrum L. & Solanaceae & Puti begun & Herb \\
\hline S. sisymbrifolium Lamk. & Solanaceae & - & Shrub \\
\hline S. torvum Swartz & Solanaceae & Gota begun & Shrub \\
\hline S. violaceum Ortega & Solanaceae & Byakur & Shrub \\
\hline S. virginianum L. & Solanaceae & Kanta kari & Shrub \\
\hline Sonneratia alba J. Smith & Sonnertiaceae & - & Tree \\
\hline S. apetala Buch.-Ham. & Sonnertiaceae & Keora & Tree \\
\hline S. caseolaris (L.) Engl. & Solanaceae & Keora & Tree \\
\hline Spatholobus acuminatus Benth. & Fabaceae & Bean & Climber \\
\hline S. roxburghii Benth. & Fabaceae & - & Climber \\
\hline Sphaeranthus indicus L. & Asteraceae & Chagalnadi & Herb \\
\hline Spilanthes acmella auct. non L. Thw. & Asteraceae & Mathamoriaguinshak & Herb \\
\hline Spondias pinnata (L.f.) Kurz. & Anacardiaceae & Amra & Tree \\
\hline Sporobolus diander (Retz.) P. Beauv. & Poaceae & Bina joni & Herb \\
\hline S. indicus R. Br. & Poaceae & - & Herb \\
\hline Stachytarpheta jamaicensis (L.) Vahl & Verbenaceae & Bina joni & Herb \\
\hline
\end{tabular}


Table 1 contd.

\begin{tabular}{|c|c|c|c|}
\hline Scientific name & Family & Bangla name & Habit \\
\hline Staurogyne argentea Wall. & Acanthaceae & - & Herb \\
\hline Stemona tuberosa Lour. & Stemonaceae & Lalgurania alu & Climber \\
\hline Stephania glabra (Roxb.) Miers & Menispermaceae & Thanda manik & Climber \\
\hline S. japonica (Thunb.) Miers & Menispermaceae & Muichanlata & Climber \\
\hline Stephegyne parvifolia Korth. auct. non Roxb. & Rubiaceae & Phulkadam & Tree \\
\hline Sterculia foetida $\mathrm{L}$. & Sterculiaceae & Jongli badam & Tree \\
\hline S. villosa Roxb. ex Smith & Sterculiaceae & Bsaket badam & Tree \\
\hline Stereospermum personatum (Hassk.) Chatterjee & Bignoniaceae & - & Tree \\
\hline Steudnera colocasioides Hook. f. & Araceae & Bishkachu & Herb \\
\hline Streblus asper Lour. & Moraceae & Sheora & Shrub \\
\hline Strobilanthes polystachia Nees.in Wall & Acanthaceae & - & Herb \\
\hline Strophanthus wallichii Decne. & Apocynaceae & - & Shrub \\
\hline Styrax serrulatus Roxb. & Styraceae & Silver bell & Shrub \\
\hline Suregada multiflora (A. Juss.) Baill. & Euphorbiaceae & Maricha & Tree \\
\hline Swietenia mahagoni Jacq. & Meliaceae & Mehogoni & Tree \\
\hline S. floribunda Griff. & Anacardiaceae & Civit & Tree \\
\hline Symplocos racemosa Roxb. & Symplocaceae & - & Climber \\
\hline Synedrella nodiflora (L.) Gaertn. & Asteraceae & - & Herb \\
\hline Syzygium balsameum (Wight) Walp. & Myrtaceae & Bhutijam & Tree \\
\hline S. claviflorum (Roxb.) A.M. Cowan \& J.M. Cowan & Myrtaceae & Nalijam & Tree \\
\hline S. cumini (L.) Skeels & Myrtaceae & Kalojam & Tree \\
\hline S. firmum Thw. & Myrtaceae & Dhakijam & Tree \\
\hline S. formosum (Wall.) Masamune & Myrtaceae & Panijam & Tree \\
\hline S. fruticosum DC. & Myrtaceae & Bhutijam & Tree \\
\hline S. syzygioides (Miq.) Merr. \& L. M. Perry & Myrtaceae & Khaijam & Tree \\
\hline Tabernaemontana corymbosa Roxb. ex Wall. & Apocynaceae & Tagar & Shrub \\
\hline T. recurvata Roxb. & Apocynaceae & Tagar & Shrub \\
\hline Tacca integrifolia Ker-Gawl. & Taccaceae & Mati munda & Herb \\
\hline Tamarindus indica L. & Campanulaceae & Tentul & Tree \\
\hline Tapiria hirsuta Hook. f & Anacardiaceae & - & Herb \\
\hline *Tectona grandis L. f. & Verbenaceae & Segun & Tree \\
\hline Tephrosia purpurea (L.) Pers. & Fabaceae & Bon-neel & Herb \\
\hline *Terminalia arjuna (Roxb. ex DC.) Wight \& Arn. & Combretaceae & Arjun & Tree \\
\hline T. bellirica (Gaertn.) Roxb. & Combretaceae & Bohera & Tree \\
\hline *T. catappa L. & Combretaceae & Katbadam & Tree \\
\hline T. chebula Retz. & Combretaceae & Haritoki & Tree \\
\hline $\begin{array}{l}\text { Tetracera sarmentosa (L.) Vahl subsp. andamanica } \\
\text { (Hoogl.) Hoogl. }\end{array}$ & Dilleniaceae & Challalata & Climber \\
\hline Tetrameles nudiflora R. Br. & Datiscaceae & Chundul & Tree \\
\hline Tetrastigma angustifolium (Roxb.) Planch. & Vitaceae & Nekung riubi & Climber \\
\hline Thunbergia fragrans Roxb. & Acanthaceae & - & Climber \\
\hline T. grandiflora (Roxb. ex Rottler) Roxb. & Acanthaceae & Nekung riubi & Climber \\
\hline
\end{tabular}


Table 1 contd.

\begin{tabular}{|c|c|c|c|}
\hline Scientific name & Family & Bangla name & Habit \\
\hline Thysanolaena maxima (Roxb.) O. Kuntze & Poaceae & Phuljharu & Herb \\
\hline Tinospora cordifolia (Willd.) Hook. f. \& Thoms. & Menispermaceae & Ghora gulancha & Climber \\
\hline T. crispa (L.) Hook. f. \& Thoms. & Menispermaceae & Gulancha & Climber \\
\hline Toona ciliata M. Roem. & Meliaceae & Toon & Tree \\
\hline Torenia vegans Roxb. & Scrophulariaceae & - & Herb \\
\hline Trema orientalis (L.) Blume & Ulmaceae & Gobar jiga & Tree \\
\hline Trevesia palmata (Roxb.) Vis. & Araliaceae & - & Shrub \\
\hline Trewia nudiflora L. & Euphorbiaceae & Pitali & Tree \\
\hline Triumfetta rhomboidea Jacq. & Tiliaceae & Banokra & Herb \\
\hline Tylophora tenuissima (Roxb.) Wight \& Arn. & Asclepiadaceae & - & Climber \\
\hline Uraria lagopoides DC. & Fabaceae & - & Herb \\
\hline U. rufesens (DC.) Schind. & Fabaceae & - & Shrub \\
\hline Urena lobata L. & Malvaceae & Banokra & Herb \\
\hline U. sinuata L. & Malvaceae & - & Herb \\
\hline Urginea indica (Roxb.) Kunth & Liliaceae & Sumudra pyaj & Herb \\
\hline Uvaria hamiltonii Hook. f. \& Thom. & Annonaceae & Latkan & Climber \\
\hline Vernonia patula (Dry) Merr. & Asteraceae & Shial lata & Herb \\
\hline Vitex altissima $\mathrm{L} . \mathrm{f}$. & Verbenaceae & Monwal & Tree \\
\hline V. trifolia L. f. & Verbenaceae & Chotonishinda & Shrub \\
\hline V. glabrata $\mathrm{R}$. Br. & Verbenaceae & Ashal & Tree \\
\hline V. peduncularis Wall. ex Schauer & Verbenaceae & Horina & Tree \\
\hline Vitis repens (Lamk.) Wight \& Arn. & Vitaceae & Marmaria puta & Climber \\
\hline Walsura robusta Roxb. & Meliaceae & Bonlichu & Tree \\
\hline Woodfordia fruticosa (L.) Kurz & Lythraceae & Dhatri-phul & Shrub \\
\hline Xanthium indicum Koen. ex Roxb. & Asteraceae & Ghagra & Herb \\
\hline Xanthophyllum flavescens Roxb. & Xanthophylaceae & Gandi & Tree \\
\hline *Xanthosoma violaceum Schott & Araceae & Dud kachu & Herb \\
\hline Zanthoxylum rhesta (Roxb.) DC. & Rutaceae & Bazna & Tree \\
\hline *Zea mays L. & Poaceae & - & Herb \\
\hline Zingiber montanum (Koen.) Dietr. & Zingiberaceae & Paletara & Herb \\
\hline Z. roseum (Roxb.) Rosc. & Zingiberaceae & Laltara & Herb \\
\hline Ziziphus funiculosa Buch.-Ham. ex Lawson & Rhamnaceae & - & Shrub \\
\hline Z. glabrata Heyne ex Roth & Rhamnaceae & Jangli kul & Climber \\
\hline *Z. mauritiana Lamk. & Rhamnaceae & Boroi & Tree \\
\hline Z. oenoplia (L.) Mill. & Rhamnaceae & Kankra & Shrub \\
\hline
\end{tabular}

The sanctuary also covered a narrow strip of mangrove vegetation at the eastern face of the hill range towards Naf river. Such vegetation is dominated by Aegialitis rotundifolia, Avicennia officinalis, Sonneratia apetala, Dalbergia spinosa and Acanthus ilicifolius. Rare occurrence of Heritiera fomes, Aegiceras corniculata and Bruguiera gymnorrhiza are also recoded in the area. A small patch of Nypa fruticans vegetation has been observed in north corner of mangrove strip vegetation. Tiger fern is also observed in the area. In the western side of hill range a special habitat called sand dune is located and the most common species are Ipomoea pes-caprae, Vitex trifolia and Pandanus foetidus. 
During the field observations and discussion with foresters and local people, we could identify a number of threats to plant diversity. These are illegal logging, political influence in illegal logging and encroachment, Rohynga pressure, brikfield in sanctuary area, cutting of plantlets for betel leaf cultivation, new road constraction, fragmentation, over ambitious foresters, unadequet manpower in the forest department in terms of forest area and minor products collections including fire wood. All such threats are enough to eliminate remaing plant diversity from the sanctuary.

For the sustainable conservation of angiosperm diversity in Teknaf wildlife sanctuary, the following recommendations have been made. Database for all species need to be made first, otherwise we will loss some of the plants before their introduction to science. Set priority for species those are sensitive to disturbance and locate them in the habitat using Greographical Information System (GIS) technique. Much effort should be given to increae their number using different propagation techniques either in situ or ex situ conditions. Effort should be made to give alternate source of income for forest products dependent people. Forest and biodiversity protection act should be implemented for over ambitious people who destroy in different ways the ability of ecosystems to support biodiversity. In 2010, the status of area has been shifted to the wildlife sanctuary because of the presence of Asian wild threatened elephant. A small part of the sanctuary has also been declared as nature Reserve Park to gain public support for biodiversity conservation. The present list of plant biodiversity (535 species) is still considered as preliminary. There might be some more species yet to be listed and few specimens remain unidentified. Based on the field observations and present preliminary results it may be concluded that the sanctuary is rich in the plant diversity and the sanctuary is the home for so many threatened plant species in context of Bangladesh. Field observation also confirmed that regeneration of tree species in the habitat is severely hampered because of anthropogenic pressure and minor product collections. Invasive species such as Urena lobata, Mikania cordata, Chromolaena odorata etc. are another challenge to future regeneration of forest trees in the disturbed areas. The study suggests for further long

term research to focus all aspects of plant biodiversity to help in making proper management plan for this sanctuary.

\section{Acknowledgement}

The paper is the outcome of the project entitled "Biodiversity monitoring in selected forests of Bangladesh" funded by Arannayk Foundation Bangladesh and implemented by the Wildlife Research Group, Department of Zoology, Jahangirnagar University. Cooperation of Forest Department during filed study is duly acknowledged.

\section{References}

Ahmed, Z.U., Begum, Z.N.T., Hassan, M.A., Khondker, M., Kabir, S.M.H., Ahmad, M., Ahmed, A.T.A., Rahman, A.K.A. and Haque, E.U. (Eds) 2008a. Encyclopedia of Flora and Fauna of Bangladesh, Vol. 6. Angiosperms: Dicotyledons (Acanthaceae - Asteraceae). Asiatic Society of Bangladesh, Dhaka, pp. 1-408.

Ahmed, Z.U., Hassan, M.A., Begum, Z.N.T., Khondker, M., Kabir, S.M.H., Ahmad, M., Ahmed, A.T.A., Rahman, A.K.A. and Haque, E.U. (Eds) 2008b. Encyclopedia of Flora and Fauna of Bangladesh, Vol. 12. Angiosperms: Monocotyledons (Orchidaceae - Zingiberaceae). Asiatic Society of Bangladesh, Dhaka, pp. 1-552.

Ahmed, Z.U., Hassan, M.A., Begum, Z.N.T., Khondker, M., Kabir, S.M.H., Ahmad, M., Ahmed, A.T.A., Rahman, A.K.A. and Haque, E.U. (Eds) 2009a. Encyclopedia of Flora and Fauna of Bangladesh, Vol. 7. Angiosperms: Dicotyledons (Balsaminaceae - Euphorbiaceae). Asiatic Society of Bangladesh, Dhaka, pp. 1-546. 
Ahmed, Z.U., Hassan, M.A., Begum, Z.N.T., Khondker, M., Kabir, S.M.H., Ahmad, M., Ahmed, A.T.A., Rahman, A.K.A. and Haque, E.U. (Eds) 2009b. Encyclopedia of Flora and Fauna of Bangladesh, Vol. 8. Angiosperms: Dicotyledons (Fabaceae - Lythraceae). Asiatic Society of Bangladesh, Dhaka, pp. 1-478.

Ahmed, Z.U., Hassan, M.A., Begum, Z.N.T., Khondker, M., Kabir, S.M.H., Ahmad, M. and Ahmed, A.T.A. (Eds) 2009c. Encyclopedia of Flora and Fauna of Bangladesh, Vol. 9. Angiosperms: Dicotyledons (Magnoliaceae - Punicaceae). Asiatic Society of Bangladesh, Dhaka, pp. 1-488.

Akter, A. and Zuberi, M.I. 2009. Invasive alien species in northern Bangladesh: Identification, inventory and impacts. International J. Biodiversity and Conservation 1(5): 129-134.

Alexiades, M.N. 1996. Selected guidelines for ethnobotanical research: A field manual. New York Botanical Garden, New York. pp. 99-133.

Bari, A. and Dutta, U. 2004. Co-management of tropical forest resources in Bangladesh. Secondary data collection for pilot protected area: teknaf Game reserve, USAID-Bangladesh and Ministry of Environment and Forest, Govt. of People's Republic of Bangladesh.

BBS (Bangladesh Bureau of Statistics) 2011. Monthly Statistical Bulletin, December 2011. Statistics Division, Ministry of Planning, Government of the People’s Republic of Bangladesh.

Choudhury, M.U. 1969. Working plan of Cox's Bazar Forest division 1968-69 to 1977-78. Forest Department, Government of East Pakistan.

Cowan, J.M. 1923. Working Plans for Cox’s Bazar Forest Division.

Cronquist, A. 1981. An Integrated System of Classification of Flowering Plants. Columbia University Press, New York.

Green, M.J.B. 1907. World Conservation Monitoring Centre IUCN Commission on National Parks and Protected Areas, Teknaf Game Reserve, IUCN Directory of South Asian Protected Areas. pp. 38-43.

Hooker, J.D. 1872-1897. The Flora of British India. Vols. 1-7. L. Reeve \& Co., Kent, England.

Hossain, M.K. and Pasha, M.K. 2004. An account of exotic flora of Bangladesh. J. Forestry and Environment 2: 99-115.

Hyland, B.P.M. 1972. A technique for collecting botanical specimens in rain forest. Flora Malesiana Bulletin 26: $2038-2040$.

Islam, M.M., Amin, A.S.M.R. and Sarker, S.K. 2003. In: Pallewatta, N., Reaser, J.K. and Gutierrer, A.T. (Eds). Invasive alien species in South-Southeast Asia. National Reports and Directory of Resources. Global Invasive Species Programme, Cape Town, South Africa, pp. 1-111.

Khan, M.S. 1990. The flora of Chunati Wildlife Sanctuary: A preliminary survey report. National Resource Information Center, Bangladesh, 31 pp.

Khan, M.S., Rahman, M.M., Huq, A.M., Mia, M.M.K. and Hassan, M.A. 1994. Assesment of biodiversity of Teknaf game reserve in Bangladesh focusing on economically and ecologically important plants species. Bangladesh J. Plant. Taxon. 1(1): 21-33.

Khan, M.S., Rahman, M.M. and Ali, M.A. (Eds). 2001. Red Data Book of Vascular Plants of Bangladesh. Bangladesh National Herbarium, Dhaka, 179 pp.

Molla, A.R., Rahman, M.M. and Rahman, M.S. 2004. Site-level field appraisal for protected area comanagement: Teknaf game reserve. Nature Conservation Movement (NACOM), Bangladesh.

Prain, D. 1903. Bengal Plants. Vols. 1 \& 2. (Ind. Repr. 1963). Botanical Survey of India, Calcutta.

Rosario, E.A. 1997. The conservation management plan of the protected areas other than those in Sundarban forest in Bangladesh. GOB/WB forest resource management project in Bangladesh.

Siddiqui, K.U., Islam, M.A., Ahmed, Z.U., Begum, Z.N.T., Hassan, M.A., Khondker, M., Rahman, M.M., Kabir, S.M.H., Ahmad, M., Ahmed, A.T.A., Rahman, A.K.A. and Haque, E.U. (Eds) 2007c. Encyclopedia of Flora and Fauna of Bangladesh, Vol. 11. Angiosperms: Monocotyledons (Agavaceae Najadaceae). Asiatic Society of Bangladesh, Dhaka, pp. 1-399.

Uddin, M.Z. and Hassan, M.A. 2004. Flora of Rema-Kalenga Wildlife Sanctuary. IUCN Bangladesh Country Office, Dhaka, Bangladesh, 120 pp. 